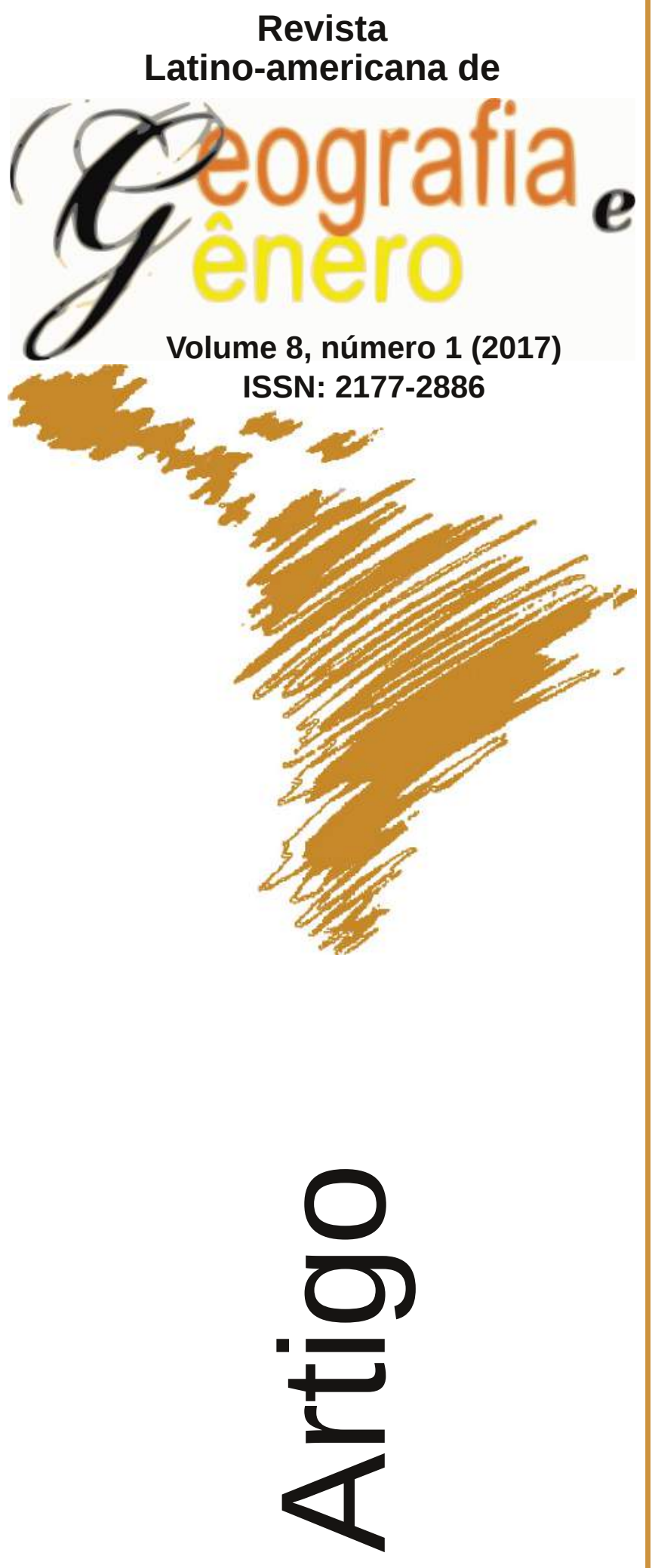

Administração Hospitalar em

Portugal, Parte II: Evidências Estatísticas de Igualdade Homem-Mulher no Envolvimento Laboral dos Enfermeiros

La Administración Hospitalaria en Portugal, Parte II: Evidencias Estadísticas de Igualdad Hombre-Mujer en la Participación Laboral de los Enfermeros

Hospital Administration in Portugal, Part II: Statistical Evidence of Man-Woman Equality in Employee Involvement of Nurses

Pedro Miguel Alves Ribeiro Correia CAPP-ISCSP, CIEG-ISCSP, Universidade de Lisboa - Portugal pcorreia@iscsp.utl.pt

Pedro Miguel Carrão Carrapato

Universidade de Lisboa - Portugal pedritocc@gmail.com

João Abreu de Faria Bilhim

CAPP-ISCSP, Universidade de Lisboa -

Portugal

bilhim@iscsp.ulisboa.pt

Como citar este artigo:

CORREIA, Pedro Miguel Alves Ribeiro; CARRAPATO, Pedro Miguel Carrão; BILHIM, João Abreu de Faria. Administração Hospitalar em Portugal, Parte II: Evidências Estatísticas de Igualdade Homem-Mulher no Envolvimento Laboral dos Enfermeiros. Revista Latino Americana de Geografia e Gênero, v. 8, n. 1, p. 64-78, 2017. ISSN 2177-2886.

Disponível em:

http://www.revistas2.uepg.br/index.php/rlagg 


\title{
Administração Hospitalar em Portugal, Parte II: Evidências Estatísticas de Igualdade Homem-Mulher no Envolvimento Laboral dos Enfermeiros
}

\author{
La Administración Hospitalaria en Portugal, Parte II: Evidencias Estadísticas \\ de Igualdad Hombre-Mujer en la Participación Laboral de los Enfermeros
}
Hospital Administration in Portugal, Part II: Statistical Evidence of Man-Woman Equality in Employee Involvement of Nurses

\section{Resumo}

Este artigo apresenta o resultado de um estudo sobre a relação entre a variável de caracterização sociodemográfica gênero e o envolvimento laboral dos enfermeiros do Hospital Beatriz Ângelo, em Portugal. A variável latente envolvimento laboral foi constituída por três variáveis de medida. Os resultados existentes na literatura sobre pesquisas que procuram relações idênticas mostram resultados discordantes. Os dados obtidos no estudo não demonstraram diferenças estatísticas significativas nos níveis de percepção sobre 0 envolvimento laboral nos enfermeiros do gênero feminino, quando comparados com os do gênero masculino, para o contexto em questão.

Palavras-Chave: Igualdade de Gênero; Administração Hospitalar; Percepções dos Colaboradores; Recursos Humanos; Envolvimento Laboral.

\section{Resumen}

En este artículo se presentan los resultados de un estudio sobre la relación entre la variable de caracterización sociodemográfica género y la participación laboral de los enfermeros del Hospital Beatriz Ângelo en Portugal. La variable latente de la participación laboral fue construida por tres variables de medida. Los resultados existentes en la literatura sobre los estudios que buscan relaciones idénticas muestran resultados contradictorios. Los datos obtenidos en el estudio no mostraron diferencias estadísticamente significativas en los niveles de percepción sobre la participación laboral en los enfermeros de género femenino, en comparación con lo de sexo masculino, para el contexto en cuestión.

Palabras-Clave: Igualdad de género; Administración Hospitalaria; Percepciones de los Empleados; Recursos Humanos; Participación Laboral.

\section{Abstract}

This article presents the results of a study on the relationship between the sociodemographic characterization variable gender and labour involvement of the nurses of Hospital Beatriz Angelo, in Portugal. The latent variable labour involvement was composed by three measurement variables. The current literature results on studies that investigate similar relationships show conflicting results. The data obtained in the study showed no statistically significant differences in perception levels of labour involvement in female nurses, when compared with male nurses, for the context in question.

Keywords: Gender Equality; Hospital Administration; Employees' Perceptions; Human Resources; Employee Involvement.

Pedro Miguel Alves Ribeiro Correia, Pedro Miguel Carrão Carrapato, João Abreu de Faria Bilhim 
Introdução

O envolvimento entre os indivíduos e as organizações onde desenvolvem a sua atividade profissional é um tema de pesquisa com pertinência não só continuada mas também crescente. As constantes mudanças organizacionais associadas a novos (e por vezes controversos) modelos de administração e/ou gestão, geram organizações cada vez mais complexas e desafios únicos, quando se procura entender os fatores impulsionadores de um maior ou menor envolvimento com as organizações por parte de quem as integra.

O consenso existente quando se discute a pertinência do tema perde-se quando se procura encontrar um conceito universal de envolvimento com as organizações. A literatura encontra explicação para esta multiplicidade de conceitos nas disciplinas que fundamentam cada um deles (BASTOS, 1993).

Paralelamente a esta multiplicidade de conceitos, crescem perspetivas teóricas distintas quando o tema do envolvimento entre os indivíduos e as organizações é abordado. Apesar da existência de autores que durante um período de tempo defenderam uma perspetiva unidimensional do envolvimento, o aprofundamento da pesquisa na área veio demonstrar que se trata de um fenômeno multidimensional (BASTOS et al., 1997).

Conscientes da multiplicidade de vetores que incorporam a relação de envolvimento que é estabelecida entre os indivíduos e as organizações, emerge a necessidade de compreender quais os fatores que conseguem influenciar as características do dinâmico processo de envolvimento. As variáveis sociodemográficas (variáveis como o gênero, idade, antiguidade, habilitações literárias, traços de personalidade ou valores) e a sua relação com o envolvimento são um dos alvos frequentes de pesquisa de âmbito internacional (CORDEIRO, 2014; SILVA, 2015).

A multiplicidade de estudos que procuram compreender a relação entre as variáveis sociodemográficas e o envolvimento tem obtido resultados contraditórios, mais concretamente quando a variável sociodemográfica considerada é o gênero (CORREIA e GARCIA, 2015).

Como contribuição para a pesquisa que procura estabelecer uma relação entre a variável sociodemográfica gênero e o envolvimento laboral, este trabalho tem como objetivo encontrar a relação entre esta variável de caracterização, gênero dos enfermeiros do Hospital Beatriz Ângelo (Loures, Portugal) e o seu envolvimento laboral. A relevância desta pesquisa é acrescida pelo fato de o Hospital Beatriz Ângelo ser uma unidade extremamente recente (inaugurado em Janeiro de 2012, o que se traduz numa avaliação do envolvimento dos enfermeiros num estado de desenvolvimento organizacional ainda bastante embrionário) e constituir um exemplo inequívoco de uma parceria público-privada, uma vez que é um dos hospitais públicos portugueses fruto de um contrato de parceria entre o Estado português e uma entidade gestora privada.

\section{A Variável Latente Envolvimento Laboral}

A pesquisa sobre o envolvimento dos trabalhadores com as organizações há muito que é desenvolvida. As constantes mudanças no mundo do trabalho

Pedro Miguel Alves Ribeiro Correia, Pedro Miguel Carrão Carrapato, João Abreu de Faria Bilhim 
tornam o tema cada vez mais relevante e premente. Mas se o consenso existe relativamente ao tema de pesquisa, desvanece-se quando se procura um conceito universal de envolvimento. Mowday, Steers e Porter fazem referência a dez conceitos diferentes de envolvimento (BASTOS, 1993).

Dos vários exemplos descritos na literatura, importa destacar Becker (1960), que define envolvimento enquanto existência de um investimento por parte de uma pessoa numa determinada área, de forma consistente, acompanhada do comprometimento com uma atividade. Medeiros, no seu trabalho de 2003, refere que Mowday, Steers e Porter definem o envolvimento como 'uma relação forte entre um indivíduo identificado e envolvido com uma organização', sendo este caracterizado por três fatores: estar disposto a exercer um esforço considerável em benefício da organização; uma forte convicção e aceitação dos objetivos da organização e um forte desejo de se manter como membro da organização (MEDEIROS et al., 2003).

Ainda segundo Bastos (1993), o conceito de Weiner e Vardi de envolvimento é definido como 'um conjunto de pressões normativas internalizadas pelo indivíduo para que se comporte congruentemente com os objetivos e interesses da organização'. Dois outros autores com trabalho realizado sobre o envolvimento, Shahnawaz e Juyal (2006) definem envolvimento como 'uma força que encaminha o comportamento dos indivíduos para realizarem um conjunto de ações relevantes para um determinado objetivo'.

Mais recentemente, Rusu (2013) afirma que existe envolvimento com a organização e os seus objetivos, quando se encontra uma 'boa vontade' em contribuir para o sucesso e alcance dos objetivos da organização, num determinado espaço e período de tempo.

A literatura justifica esta multiplicidade de conceitos com a base teórica que os sustenta. Bastos (1993) identifica três áreas científicas como principais fontes de pesquisa sobre o envolvimento organizacional: a sociologia, as teorias organizacionais e a psicologia social.

Weber e Becker são dois dos autores que estão associados ao estudo do envolvimento com as organizações na área da sociologia. Enquanto Weber contribui com o seu trabalho no campo da teoria das organizações, mais especificamente por intermédio das ciências da administração, Becker constrói o conceito de envolvimento instrumental, onde associa a permanência do indivíduo na organização aos custos e benefícios associados á sua saída (MEDEIROS et al., 2003).

Das teorias organizacionais evolui o conceito de envolvimento afetivo ou atitudinal, que dominou a literatura por um considerável período de tempo. Este surge com trabalhos desenvolvidos por autores como Mowday, Steers e Porter, que apesar de reconhecerem a existência da perspetiva comportamental, defendem que o envolvimento com a organização é mais do que uma ligação passiva. Acreditam que o envolvimento é uma ligação ativa onde, para além da noção de identificação com a organização, existe associação com as dimensões de lealdade, com o desejo em permanecer na organização e de empreender esforços em benefício da organização (BASTOS 1993; MEDEIROS et al., 2003).

Por seu turno, a psicologia social contribui com a dimensão normativa e 
comportamental. Wiener (1982) é usualmente mencionado quando se aborda a questão da aceitação dos objetivos e valores da organização enquanto forma de controlo das ações dos indivíduos. Quando envolvidos com a organização, os colaboradores demonstram determinados comportamentos por serem moralmente corretos (forma de controlo denominada de normativoinstrumental) (MEDEIROS et al., 2003).

Destacam-se duas perspetivas teóricas relativamente ao envolvimento organizacional. Uma baseada em modelos unidimensionais, com uma abordagem mais direta e um conceito livre de tensões, onde o envolvimento é considerado como um vínculo afetivo pela ligação entre o indivíduo e a organização; e outra que surge a partir da observação das diferenças e semelhanças que existem nos vários conceitos unidimensionais de envolvimento organizacional (FREITAS, 2010).

A maior parte da pesquisa sobre o tema do envolvimento organizacional tem como base modelos unidimensionais relativamente à sua definição e medida do conceito. A descrição da natureza do estado psicológico serve de base para a relação entre a organização e o indivíduo. A perspetiva que domina a pesquisa do envolvimento organizacional tem por base a abordagem afetiva ou atitudinal. Os indivíduos permanecem nas organizações com base no desenvolvimento de uma relação afetiva. Identificam-se, envolvem-se com a organização e desejam permanecer nesta como forma de colaborar no alcance dos objetivos estabelecidos, com base no desenvolvimento de uma relação afetiva (BASTOS, 1993; ROWE, 2008).

A abordagem instrumental é outra das perspetivas unidimensionais descritas na literatura. Os seus proponentes defendem que o envolvimento dos indivíduos está relacionado com o reconhecimento dos custos e/ou benefícios que implica a sua saída da organização (MEDEIROS et al., 2003).

A terceira perspetiva unidimensional descrita é a abordagem normativa. Esta associa o envolvimento a um dever moral de permanecer na organização. Existe, por parte do indivíduo, uma ligação com a organização através dos objetivos, missão e valores, o que transfere para o sujeito o dever moral de permanecer na organização. Segundo esta perspetiva, o envolvimento pode levar os indivíduos a terem determinado comportamento, não porque 0 entendam como benéfico para eles próprios, mas porque o entendem como o comportamento moralmente correto (BASTOS, 1993; ROWE, 2008).

Não obstante, a recorrente pesquisa sobre o envolvimento laboral avolumou evidências no sentido de que se trata de um fenômeno multidimensional. Esta linha de pensamento pretende identificar as várias variáveis que integram e concorrem para a formação das percepções sobre o envolvimento. Estudos específicos sobre a dimensionalidade das medidas de envolvimento, em conjunto com estudos que procuram melhorar os modelos que explicam outros fenômenos organizacionais, tornam mais consistentes as tentativas de particionar o conceito de envolvimento com as organizações. Tal traduz-se no primeiro passo para a construção de diferentes tipologias de envolvimento (BASTOS et al., 1997).

A perspetiva multidimensional do envolvimento pode ser encarada como uma efetiva conquista conceitual. Meyer e Allen são os autores do modelo multidimensional que mais consenso reúne atualmente, conhecido como o

Pedro Miguel Alves Ribeiro Correia, Pedro Miguel Carrão Carrapato, João Abreu de Faria Bilhim 
Modelo de Conceitualização de Três Componentes do Comprometimento Organizacional (RUSU, 2013). Segundo este modelo, o envolvimento dos indivíduos com as organizações é constituído por três componentes: afetivo (affective), de continuidade (continuance) e normativo (normative). A pesquisa tornou claro que é comum a estas três abordagens a leitura do envolvimento enquanto estado psicológico que caracteriza a relação do indivíduo com a organização, com implicações na sua decisão de continuar ou não a pertencer à mesma (MEDEIROS, et al. 2003).

O envolvimento afetivo corresponde ao apego, identificação e envolvimento que o indivíduo tem com a organização, pelo que o mesmo é dizer que os indivíduos com forte envolvimento afetivo estabelecem uma relação emocional e permanecem nela porque o querem fazer. O envolvimento de continuidade (ou instrumental) está relacionado com os custos percebidos pelos indivíduos em deixar a organização, isto é, os indivíduos permanecem na organização porque precisam. $\mathrm{O}$ envolvimento normativo está relacionado com o sentimento de obrigatoriedade em pertencer à organização, ou seja, os indivíduos permanecem na organização por entenderem que devem permanecer (MEDEIROS, et al. 2003).

A literatura refere ser expectável que os indivíduos experimentem as três diferentes formas de envolvimento com diferentes níveis de intensidade. Um indivíduo pode sentir um desejo e uma necessidade forte em manter-se na organização e pouca obrigação de o fazer (forte componente afetiva e instrumental, e fraca componente normativa). Também pode sentir pouco desejo de permanecer, uma necessidade moderada e uma forte obrigação em pertencer na organização (fraca componente afetiva, moderada componente instrumental e forte componente normativa). Desta forma, torna-se evidente a vantagem desta perspetiva multidimensional, que consiste em esperar que os vários tipos de envolvimento interajam entre si e influenciem o comportamento dos indivíduos (MEYER e ALLEN, 1991).

Um outro modelo multidimensional descrito na literatura é o Modelo do Vinculo Psicológico do Empregado, desenvolvido por O'Reilly e Chatman. Os autores desenvolveram o seu modelo baseado nos trabalhos sobre atitudes e alterações de comportamentos de Kelman (1958), que identificou um vínculo psicológico com as organizações baseado em três bases independentes. A submissão ou o envolvimento instrumental (as atitudes e comportamentos são motivados por recompensas exteriores), a identificação (o indivíduo deixa-se influenciar por um desejo de manutenção de relações satisfatórias) e internalização (as atitudes e comportamentos são motivados pela congruência entre os valores individuais e organizacionais) (MEDEIROS, et al. 2003).

Neste artigo será utilizada uma abordagem multidimensional do envolvimento laboral e estudada a sua relação com a variável de caracterização sociodemográfica gênero, tópico abordado em detalhe no próximo ponto do texto.

\section{Variável de Caracterização Sociodemográfica Gênero e a sua Relação com o Envolvimento Laboral}

A necessidade de compreender a relação entre o envolvimento laboral no

Pedro Miguel Alves Ribeiro Correia, Pedro Miguel Carrão Carrapato, João Abreu de Faria Bilhim 
seio das organizações fez crescer o número de trabalhos que procuram estabelecer relações entre os fatores que influenciam o envolvimento e as consequências que este pode ter dentro de uma organização. Apesar da divergência e contradição ao nível dos resultados das pesquisas que relacionam o envolvimento nas organizações é consensual, entre diversos autores, o carater dinâmico do processo de envolvimento, o reconhecimento da sua influência na persecução dos objetivos organizacionais e a ausência de ligação direta inequívoca entre envolvimento dos colaboradores e sucesso da organização (BASTOS, 1993; MEDEIROS, 2003; MEDEIROS et al., 2003).

A relação entre as variáveis sociodemográficas e o envolvimento na organização é uma das relações alvo da pesquisa internacional na área. Meyer e Allen (1997) propõem um modelo que divide o estudo das causas do envolvimento organizacional em três modelos principais: modelos causais, modelos processuais e modelos de políticas e práticas de recursos humanos. Dentro do primeiro estão incluídos os estudos relacionados com variáveis sociodemográficas (variáveis como o gênero, idade, antiguidade, habilitações literárias, traços de personalidade ou valores) e as características do posto de trabalho ou função (autonomia, conteúdo da função, diversidade das atividades, segurança no trabalho, entre outros) (CORDEIRO, 2014; SILVA, 2015).

As referências na relação entre o gênero e o envolvimento com as organizações são contraditórias. Correia e Garcia (2015) referem a ausência de evidências significativas, apresentada em alguns estudos, entre o gênero e o envolvimento no trabalho, a que contrapõem outros estudos onde foram encontrados níveis superiores de envolvimento nos indivíduos de gênero masculino quando comparados com o gênero feminino.

Um dos trabalhos frequentemente referido quando o tema de discussão é a relação entre o envolvimento laboral e as características sociodemográficas, é a meta-análise de Mathieu e Zajac (1990), onde foram apresentadas fortes evidências de que variáveis como idade, gênero, estado civil, número de filhos, percepção de alternativas de trabalho e sistemas de pagamento têm, em geral, um papel modesto no incremento do envolvimento com a organização (BASTOS, 1993; BORGES-ANDRADE, 1994). Meyer e Allen (1991) no seu trabalho A Three-Component Conceptualization of Organizational Commitment também apontam uma relação fraca e pouco consistente entre as características pessoais e o envolvimento. Estes autores referem que foi demonstrado que a relação entre as caraterísticas sociodemográficas e o envolvimento tende a desvanecer quando os prêmios de desempenho e os valores organizacionais entram na equação.

A meta-análise de Kibeom et al. (2000), pelo contrário, sugere que o gênero feminino tende a ser mais envolvido no trabalho do que o gênero masculino, pela maior quantidade de obstáculos que as mulheres têm que superar para entrar no mundo do trabalho.

Quando o foco incide nas organizações do setor da saúde, particularmente no que diz respeito à compreensão do complexo processo de retenção dos profissionais de saúde, em geral, e dos enfermeiros, em particular, bem como as suas consequências e a compreensão dos fatores que o moderam e desencadeiam pode resultar em benefícios para os indivíduos e para as 


\section{Metodologia}

A componente empírica deste estudo teve por base o inquérito por questionário de 351 enfermeiros do Hospital Beatriz Ângelo, hospital público, integrado no Serviço Nacional de Saúde de Portugal, que resultou do contrato de parceria entre uma entidade gestora privada e o Estado Português. Os dados foram colhidos no decorrer do mês de dezembro de 2015. Do total das 356 respostas obtidas, 351 foram consideradas válidas para a variável de caracterização gênero. Num universo de 624 enfermeiros, as 351 respostas obtidas configuram uma taxa de resposta de $56,25 \%$, valor consideravelmente superior ao obtido usualmente em estudos de natureza similar.

Para a variável de caracterização gênero relativamente aos enfermeiros inquiridos, foram definidas as categorias usuais: feminino e masculino. A variável latente analisada foi a percepção do envolvimento atual com a atividade profissional dos enfermeiros. A constituição da variável latente foi realizada com recurso a três variáveis de medida ${ }^{1}$ :

- Orgulho que tem em pertencer a esta organização (conceito particular: orgulho);

- Dedicação e empenho que tem pela organização (conceito particular: dedicação e empenho);

- Grau em que está disposto a sacrificar o seu tempo pessoal para

1 De forma a compreender mais aprofundadamente a construção da variável latente envolvimento com a atividade profissional deve ser consultado Correia (2012).

Pedro Miguel Alves Ribeiro Correia, Pedro Miguel Carrão Carrapato, João Abreu de Faria Bilhim 
concluir o seu trabalho (conceito particular: sacrifício).

Para as três variáveis de medida definidas, integrantes da variável latente, utilizaram-se escalas por intervalo, numéricas, de Likert com 10 pontos, limitadas nos extremos (limite inferior: 'nível muito baixo'; limite superior: 'nível muito elevado'), e com a possibilidade adicional de resposta 'não sabe/não responde'.

Pela ausência de normalidade da distribuição dos níveis das percepções sobre o envolvimento laboral em cada uma das categorias de gênero (uma das condições obrigatórias para a utilização do teste de igualdade das médias) foi necessário recorrer ao teste não paramétrico de Mann-Whitney ${ }^{2}$ (MANN e WHITNEY, 1947), para determinar as relações estatísticas existentes nos dados. A aplicação do teste possibilitou a produção de afirmações, com legitimidade estatística, sobre a ocorrência de valores mais altos dos níveis de percepção sobre o envolvimento laboral dos enfermeiros do gênero masculino, quando comparados com os níveis de percepção das enfermeiras do gênero feminino, sobre o seu inverso, ou ainda sobre a inexistência de desigualdades a este nível. Para a aplicação de qualquer dos testes estatísticos referidos, o nível de significância definido foi de $0,05(5,00 \%)$.

\section{Resultados}

Na Tabela 1 encontram-se os resultados das 351 observações consideradas válidas. Destas, 263 correspondem a colaboradores do gênero feminino $(74.9 \%)$ e 88 observações a colaboradores do gênero masculino $(25,1 \%)$.

Tabela 1 - Numero de observações por categoria de gênero e respetivo peso percentual.

\begin{tabular}{|c|c|c|}
\hline Gênero & Número de observações & Porcentagem do Total \\
\hline Feminino & 263 & $74,9 \%$ \\
\hline Masculino & 88 & $25,1 \%$ \\
\hline Total & 351 & $100,0 \%$ \\
\hline
\end{tabular}

No Gráfico 1 estão representados os valores obtidos para a variável latente (envolvimento laboral) e para as variáveis de medida que a constituem. De forma global, nas 351 observações, o valor médio obtido para o nível da percepção sobre o envolvimento laboral foi de 7,02 pontos (mediana de 7,17 pontos), o que corresponde a um nível elevado (entre 5 e 8 pontos). O valor foi obtido com base nas avaliações médias apuradas para cada uma das variáveis

2 Os testes estatísticos não paramétricos baseiam-se na distribuição dos valores das variáveis. Por essa razão são testes que não recorrem a nenhum parâmetro em particular (como por exemplo a média ou a proporção). A aplicação do teste de Mann-Whitney (pela ausência, nos subgrupos do gênero feminino e do gênero masculino, das propriedades matemáticas apropriadas para comparar as suas médias) permite considerar estatisticamente a possibilidade de os dados associados a cada um dos gêneros pertencerem à mesma população ou se, pelo contrário, se deve presumir que derivam de populações diferenciadas. Um exemplo de aplicação do teste de Mann-Whitney pode ser encontrado, por exemplo, em Correia e Videira (2015).

Pedro Miguel Alves Ribeiro Correia, Pedro Miguel Carrão Carrapato, João Abreu de Faria Bilhim 


\section{Administração Hospitalar em Portugal, Parte Il: Evidências Estatísticas de}

Igualdade Homem-Mulher no Envolvimento Laboral dos Enfermeiros

de medida, levando em consideração os respetivos pesos normalizados ${ }^{3}$ :

- $\quad$ Orgulho que tem em pertencer a esta organização (média de 6,9 peso normalizado de $45,0 \%$ );

- Dedicação e empenho que tem pela organização (média de 7,9; peso normalizado de $26,7 \%$ );

- Grau em que está disposto a sacrificar o seu tempo pessoal para concluir o seu trabalho (média de 6,4; peso normalizado de 28,4\%).

Gráfico 1 - Variável latente envolvimento laboral e respetivas variáveis de medida constituintes.

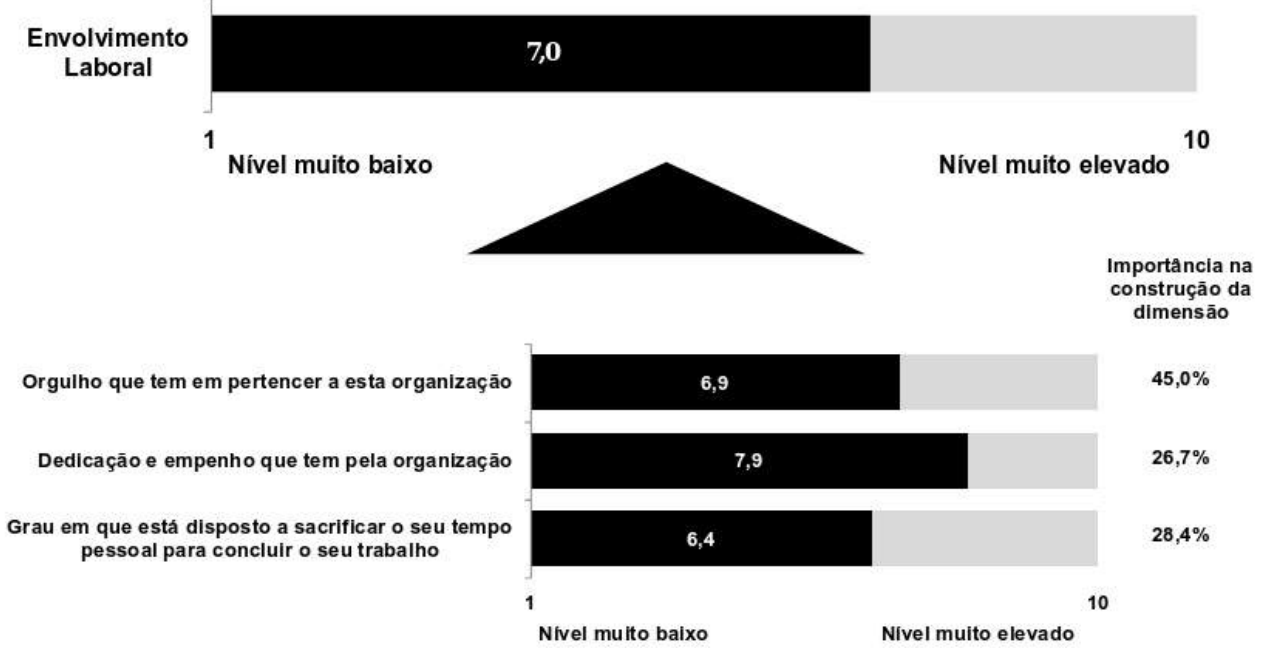

A confiabilidade do questionário quando se recorre a variáveis latentes deve ser tida em consideração. Há que garantir que as questões são convergentes (todas as questões medem o mesmo fenômeno), discriminantes (garantem a capacidade de distinguir diferentes aspetos do mesmo fenômeno) e que verificam a unidimensionalidade da variável latente definida (ausência de necessidade de subdividir a variável latente em duas ou mais categorias). A análise efetuada permitiu verificar a adequação das variáveis de medida utilizadas e alta confiabilidade do questionário realizado ${ }^{4}$.

O gráfico 2 apresenta os valores obtidos para as médias e medianas dos níveis de percepção dos enfermeiros face ao envolvimento laboral, segundo o gênero do respondente.

3 Para obter uma explicação mais pormenorizada sobre a construção da variável latente deve ser consultado Correia (2012); para aplicações semelhantes desta metodologia, consulte-se Correia (2013), Correia et al. (2013), Correia e Bilhim (2014) ou Correia e Garcia (2015).

4 A verificação da unidimensionalidade foi tratada com recurso ao alpha de Cronbach, do ró de Dillon-Goldstein e do critério de Kaiser aplicado aos valores próprios normalizados das componentes principais resultantes de uma análise fatorial aos indicadores da variável latente. Os estudos para validação de convergência e para validação de descriminação de forma a verificar e complementar a confiabilidade das variáveis de medida tiveram como base o conceito de Average Variance Explained (AVE) (TENENHAUS et al., 2005). A metodologia utilizada pode ser consultada de forma detalhada em Correia (2012).

Pedro Miguel Alves Ribeiro Correia, Pedro Miguel Carrão Carrapato, João Abreu de Faria Bilhim 
Administração Hospitalar em Portugal, Parte II: Evidências Estatísticas de

Igualdade Homem-Mulher no Envolvimento Laboral dos Enfermeiros

Gráfico 2 - Valores dos níveis de percepção dos enfermeiros face envolvimento laboral, segundo o gênero.

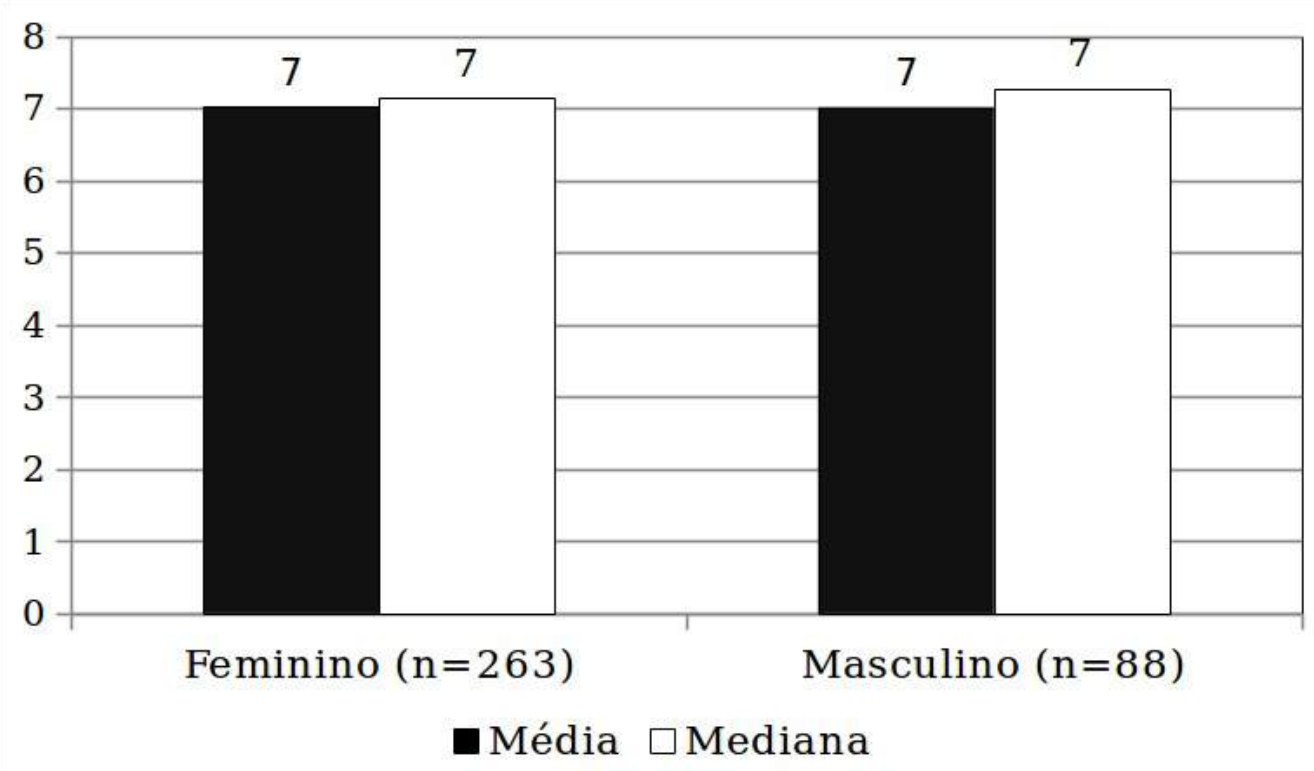

O valor médio obtido pelas mulheres de 7,02 pontos apresenta uma diferença positiva, de 0,01 , em relação aos homens. Estes apresentam um valor médio de 7,01 pontos (ambos com níveis elevados). Relativamente à mediana, é possível observar que as mulheres apresentam uma mediana de 7,15 pontos, registando uma diferença negativa de 0,13 pontos, em relação aos homens, que apresentam uma mediana de 7,28 pontos. O teste não paramétrico de MannWhitney foi aplicado com o objetivo de aferir se estas diferenças são estatisticamente significativamente ou se podem ser atribuídas a perturbações aleatórias dos dados.

Os resultados obtidos da aplicação do teste não paramétrico de MannWhitney encontram-se na Tabela 2. De destacar o p-valor aferido de 0,798. Tendo por referência o nível de significância estabelecido anteriormente $(0,05)$, é possível constatar que não existem evidências estatísticas que possibilitem a rejeição da hipótese nula do teste. Por outras palavras, não foram encontradas diferenças estatisticamente significativas nos valores dos níveis de percepção sobre o envolvimento laboral, em função do gênero dos enfermeiros.

Tabela 2 - Resultados do teste de Mann-Whitney para a variável envolvimento laboral, agrupada segundo o gênero.

\begin{tabular}{|c|c|}
\cline { 2 - 2 } \multicolumn{1}{c|}{} & Sistema de Avaliação \\
\hline Valor do teste Mann-Whitney & 11361,500 \\
\hline Valor de Z & -0.256 \\
\hline p-valor (bicaudal) & 0.798 \\
\hline
\end{tabular}

Pedro Miguel Alves Ribeiro Correia, Pedro Miguel Carrão Carrapato, João Abreu de Faria Bilhim 


\section{Discussão e Conclusões}

A pesquisa que estuda a relação entre as variáveis sociodemográficas e o envolvimento com as organizações não apresenta resultados convergentes. Enquanto alguns autores apresentam evidências no sentido da existência de relações significativas entre o gênero e o grau de envolvimento (KIBEOM et al., 2000) (sem conseguirem, no entanto, compreender o motivo pelo qual esta diferença acontece), outros referem ausência de relação entre o gênero e o envolvimento laboral (CORREIA e GARCIA, 2015).

Os dados obtidos ao longo do presente estudo contribuem para o avolumar de evidências no sentido da não existência de diferenças estatísticas significativas nos níveis de percepção sobre do envolvimento laboral em enfermeiros do gênero feminino, quando comparados com os do gênero masculino (medianas de 7,15 versus 7,28, respetivamente). Conclui-se que a variável sociodemográfica gênero dos enfermeiros do Hospital Beatriz Ângelo não apresenta relação com o seu nível de envolvimento laboral.

Para além do exposto, é possível extrair conclusões sobre a variável latente e as suas variáveis de medida constituintes. Globalmente, o nível da percepção do envolvimento obtido foi um nível elevado. Analisadas as variáveis de medida individualmente, constata-se que tal acontece pelo valor obtido na variável de medida dedicação e empenho. Na literatura, é possível encontrar referências no sentido de que o envolvimento dos enfermeiros pode ser um fator moderador (entre outros) da sua intenção de saída ou permanência na organização (HAYES et al., 2006), algo que se reveste de extrema importância para a administração do hospital em questão.

Sugere-se como trabalho futuro a aplicação do mesmo instrumento de recolha de dados a outros grupos profissionais dentro da mesma organização, de forma a estabelecer a existência ou ausência de relação entre a variável gênero e o grupo profissional. Pode ser interessante a replicação do estudo de forma mais alargada, com a aplicação do mesmo instrumento de recolha de dados e modelo de análise em hospitais portugueses com modelos de gestão diferentes e em hospitais de outros países com língua oficial portuguesa, de modo a tornar os resultados reportados neste artigo mais robustos e abrangentes.

Apesar da ausência de relação entre o envolvimento laboral e o gênero dos enfermeiros, observada no contexto desta pesquisa, a existência de uma relação positiva entre o envolvimento e o processo de socialização dos enfermeiros, o estudo detalhado do mecanismo pelo qual o envolvimento influencia o turnover, e a ligação destas temáticas a um eventual esbatimento daquilo que na literatura é conhecido como paradox of the contented working woman (TOLBERT e MOEN, 1998; CORREIA e GARCIA, 2015), em particular no setor da saúde, abre caminho para relevantes investigações futuras. 
BASTOS, Antonio. Comprometimento Organizacional: Um Balanço dos Resultados e Desafios que Cercam essa Tradição de Pesquisa. Revista de Administração de Empresas, v. 33, n. 3, p. 52 - 64, 1993.

BASTOS, Antonio; BRANDÃO, Margarida; PINHO, Ana. Comprometimento Organizacional: Uma Análise do Conceito Expresso por Servidores Universitários no Cotidiano de Trabalho. Revista de Administração Contemporânea, v. 1, n. 2, p. 97 - 120, 1997.

BECKER, Howard. Notes on the Concept of Commitment. American Journal of Sociology, v. 66, n. 1, p. 32 - 40, 1960.

BORGES-ANDRADE, Jairo. Conceituação e Mensuração de Comprometimento Organizacional. Temas em Psicologia, v. 2, n. 1, p. 37 - 47, 1994.

CORDEIRO, João. Comprometimento Organizacional: O Caso dos Docentes de uma Instituição de Ensino Superior. In: XXIV JORNADAS LUSOESPANHOLAS DE GESTÃO CIENTÍFICA, 2014, Leiria.

CORREIA, Pedro. Igualdade de Gênero no Ministério da Justiça em Portugal: Evidências Estatísticas de Igualdade Homem-Mulher na Lealdade Laboral. Direitos Fundamentais \& Justiça, v. 7, n. 23, p. 121 - 130, 2013.

CORREIA, Pedro. O Impacto do Sistema Integrado de Gestão e Avaliação do Desempenho da Administração Pública (SIADAP) na Satisfação dos Colaboradores - O Caso dos Serviços do Ministério da Justiça em Portugal. 2012. Tese (Doutoramento em Ciências Sociais na Especialidade de Administração Pública) - Universidade Técnica de Lisboa, Lisboa.

CORREIA, Pedro; BILHIM, João. A antiguidade na organização e a satisfação laboral dos colaboradores do Ministério da Justiça em Portugal: evidências de uma relação em forma de L e não em forma de U. Revista de Economia e Administração, v. 13, n. 2, p. 159 - 177, 2014.

CORREIA, Pedro; GARCIA, Bruno. Administração Hospitalar em Portugal: Evidências Estatísticas de Igualdade Homem-Mulher nas Percepções sobre os Sistemas de Avaliação de Desempenho. Revista Latino-americana de Geografia e Gênero, v. 6, n. 1, p. 127 - 139, 2015.

CORREIA, Pedro; MOREIRA, Maria; GARCIA, Bruno. Igualdade de Gênero no Ministério da Justiça em Portugal: Evidências Estatísticas de Diferenças Homem-Mulher na Satisfação Laboral. Scientia Ivridica, v. 62, n. 333, p. 569 - 590, 2013.

CORREIA, Pedro; VIDEIRA, Susana. Troika's Portuguese Ministry of Justice Experiment: An Empirical Study on the Success Story of the Civil Enforcement Actions. International Journal for Court Administration, v. 7, 
Administração Hospitalar em Portugal, Parte II: Evidências Estatísticas de

Igualdade Homem-Mulher no Envolvimento Laboral dos Enfermeiros

n. 1, p. 37 - 50, 2015.

FERREIRA, Maria; SILVA, Isilda. Empenhamento Organizacional de Enfermeiros e Relação com a Chefia. Revista Enfermagem UERJ, v. 21, n. 2, p. $156-161,2013$.

FREITAS, Maria. Os Níveis de Comprometimento em Contexto Organizacional: Estudo de Uma Empresa Portuguesa. 2010. Dissertação (Mestrado em Ciências Empresariais) - Universidade Fernando Pessoa.

HAYES, Lauren; O'BRIEN-PALLAS, Linda; DUFFIELD, Christine; SHAMIAN, Judith; BUCHAN, James; HUGHES, Frances; LASCHINGER, Heather; NORTH, Nicola; STONE, Patricia. Nurse Turnover: A Literature Review. International Journal of Nursing Studies, v. 43, n. 2, p. 237 - 263. 2006.

HAYES, Lauren; O'BRIEN-PALLAS, Linda; DUFFIELD, Christine; SHAMIAN, Judith; BUCHAN, James; HUGHES, Frances; LASCHINGER, Heather; NORTH, Nicola. Nurse Turnover: A Literature Review - An Update. International Journal of Nursing Studies, v. 49, n. 7, p. 887 - 905, 2012.

KELMAN, Herbert. "Compliance, Identification, and Internalization: Three Processes of Attitude Change". Journal of Conflict Resolution, vol. 2, n. ${ }^{\circ}$, pp. 51-60, 1958.

KIBEOM, Lee; CARSWELL, Julie; ALLEN, Natalie. A Meta-Analytic Review of Occupational Commitment: Relations with Person and WorkRelated Variables. Journal of Applied Psychology, v. 85, n. 5, p. 799 - 811, 2000 .

LU, Kuei; LIN, Pi-Li; WU, Chiung-Man; HSIEH, Ya-Lung; CHANG, YongYuan. The Relationships among Turnover Intentions, Professional Commitment, and Job Satisfaction of Hospital Nurses. Journal of Professional Nursing, v. 18, n. 4, p. 214 - 219, 2002.

MANN, Henry; WHITNEY, Donald. On a Test of Whether One of Two Random Variables is Stochastically Larger than the Other. Annals of Mathematical Statistics, v. 18, n. 1, p. 50-60, 1947.

MATHIEU, John; ZAJAC, Dennis. A Review and Meta-Analysis of the Antecedents, Correlates, and Consequences of Organizational Commitment. Psychological Bulletin, v. 108, n. 2, p. 171 - 194, 1990.

MEDEIROS, Carlos. Comprometimento Organizacional: Um Estudo de Suas Relações com Características Organizacionais e Desempenho nas Empresas Hoteleiras. 2003. Tese (Doutoramento em Administração de Empresas) - Universidade de São Paulo, São Paulo.

MEDEIROS, Carlos; ALBUQUERQUE, Lindolfo; SIQUEIRA, Michella; MARQUES, Glenda. Comprometimento Organizacional: O Estado da Arte da 
Administração Hospitalar em Portugal, Parte II: Evidências Estatísticas de

Igualdade Homem-Mulher no Envolvimento Laboral dos Enfermeiros

Pesquisa no Brasil. Revista de Administração Contemporânea, v. 7, n. 4, p. 187 - 209, 2003.

MEYER, John; ALLEN, Natalie. A Three-Component Conceptualization of Organizational Commitment. Human Resource Management Review, v. 1, n. 1, p. 61 - 89, 1991.

MEYER, John; ALLEN, Natalie. Commitment in the Workplace: Theory, Research, and Application. London, United Kingdom: SAGE Publications, 1997.

TENENHAUS, Michel; VINZI, Vincenzo; CHATELIN, Yves-Marie; LAURO, Carlo. PLS Path Modeling. Computational Statistics and Data Analysis, v. 48, n. 1, p. 159 - 205, 2005.

REID, Carol; HURST, Cameron; ANDERSON, Debra. Examination of SocioDemographics and Job Satisfaction in Australian Registered Nurses. Collegian, v. 20, n. 3, p. 161 - 169, 2013.

ROWE, Diva. Múltiplos Comprometimentos e Suas Relações com o Desempenho: Um Estudo entre Docentes do Ensino Superior Brasileiro em IES Públicas e Privadas. 2008. Tese (Doutoramento em Administração) Universidade Federal da Baia.

RUSU, Raluca. Organizational Commitment - From its Beginnings Until Today. Scientific Bulletin, v. 18, n 2, p. 181 - 186, 2013.

SHAHNAWAZ, Ghazi; JUYAL, Rakech. Human Resource Management Practices and Organizational Commitment in Different Organizations. Journal of the Indian Academy of Applied Psychology, v. 32, n. 3, p. 171 - 178, 2006.

SILVA, Cláudia. Gestão de Recursos Humanos e Comprometimento Organizacional: O Caso das USF. 2015. Dissertação (Mestrado em Gestão Estratégica De Recursos Humanos) - Escola Superior de Ciências Empresariais.

TOLBERT, Pamela; MOEN, Phyllis. Men's and Women's Definitions of 'Good' Jobs. Work \& Occupations, v. 25, n. 2, p. 168 - 195, 1998.

WIENER, Yoash. Commitment in Organizations: A Normative View. Academy Of Management Review, v. 7, n. 3, p. 418 - 428, 1982.

WAUGAMAN, Wynne; LOHRER, Donna. From Nurse to Nurse Anesthetist: The Influence of Age and Gender on Professional Socialization and Career Commitment of Advanced Practice Nurses. Journal of Professional Nursing, v. 16, n. 1, p. $47-56,2000$.

Recebido em 10 de fevereiro de 2016. Aceito em 09 de setembro de 2016.

Pedro Miguel Alves Ribeiro Correia, Pedro Miguel Carrão Carrapato, João Abreu de Faria Bilhim 